\title{
The effect of atmospheric attenuation on inclined cosmic ray air showers
}

\author{
M.T. Dova, L.N. Epele, A.G. Mariazzi * \\ Department of Physics, Universidad Nacional de La Plata, C.C. 67-1900 La Plata, Argentina
}

Received 11 October 2001; accepted 10 May 2002

\begin{abstract}
The increasing cosmic ray statistics collected by present experiments and the future prospects with new large arrays demand accurate calculations of the extensive air shower (EAS) parameters. The energy of the primary particle is estimated by ground arrays fitting a lateral distribution function (LDF) to the particle densities at a given observing level. However, the lack of appropriate parameterization for these distributions, able to reproduce the data collected from all arrival directions, makes the experimental analysis difficult. We propose a method to parametrize particle density distributions of EAS at any incident zenith angle. Starting from analytical LDF for vertical showers we present a detailed study of the atmospheric depth dependence of the shower parameters. The results obtained are used to calculate the corresponding LDF for non-vertical showers including for the first time both, geometrical and atmospheric attenuation effects. We check the method analysing electron and muon LDF generated by Monte Carlo simulations from incident cosmic ray particles at different zenith angles. A comparison of the proposed LDF with experimental results, as well as MC data including detector effects, is also presented.
\end{abstract}

(c) 2002 Elsevier Science B.V. All rights reserved.

PACS: $6.40 . \mathrm{Pq} ; 96.40$

Keywords: Cosmic rays; Extensive air showers; Lateral distribution functions

\section{Introduction}

The observation of cosmic ray particles with energies well beyond the predicted GreisenZatsepin-Kuzmin cutoff [1-4], enhanced by the discrepancies in the cosmic ray spectrum recently reported by HiRes and AGASA experiment [4,5],

\footnotetext{
${ }^{*}$ Corresponding author. Tel.: +54-221-4246062; fax: +54221-4252006.

E-mail address: mariazzi@fisica.unlp.edu.ar (A.G. Mariazzi).
}

is pushing astroparticle physics into a period of fast development both theoretically and experimentally.

The analysis of the extensive air showers (EAS) produced when the high energy particle enters in the atmosphere and interacts with the air molecules is crucial to know the characteristics of the ultrahigh energy cosmic rays (UHECR). On the other hand, studies of the high energy interaction taking place in the atmosphere could yield unique information for elementary particle physics.

The interest in EAS studies has been revived mainly because improvements achieved with new 
large arrays and the increasing statistics collected demand accurate calculations of the shower parameters. A very important shower observable is the spatial distribution of particles at a given observing level because ground array techniques use the particle density distributions of EAS to estimate the energy of the primary particle, fitting a lateral distribution function (LDF) to the observed particle densities [6]. There exist several LDF parameterizations well suited to describe density distributions for primary incident angles smaller than $45^{\circ}$, which are currently used by cosmic ray experiments.

High zenith angle showers induced by protons, heavy nuclei or photons have been subject of recent studies as an important tool to obtain relevant information about $\mathrm{CR}$ mass composition $[7,8]$. Very inclined showers are also of great importance since they are the main background for neutrino detection as expected in the Pierre Auger Observatory [9-11]. Although CR experiments have detected showers with large zenith angles, most of these data have not been yet analyzed due to the difficulties present in understanding the space-time structure of the inclined showers. It is worth mentioning that the event reconstruction is performed in all air shower arrays assuming that the observation depends only on perpendicular distance from the shower axis. This very strong assumption, if not satisfied, may introduce important errors in core position and energy determination; precisely this is the case for large zenith angle showers. The main difference in analyzing inclined showers lies in the fact that the assumed circular symmetry for the observed particle densities from vertical showers is broken. Besides, there is an asymmetry due to the different path traveled by particles in the upper and lower sides of the plane perpendicular to the shower axis (attenuation effect). These effects which are small at $\theta<45^{\circ}$ become important at larger zenith angles, producing a significant asymmetry of the lateral distribution of particle density. On the other hand, the charged particle trajectories through the atmosphere are distorted in the geomagnetic field, effect extensively studied [12,2,13-15]. A recent analysis of the influence of the magnetic field of the Earth on the shower observables for the southern site for the Pierre Auger Observatory (average geomagnetic field of 25,000 nT and site altitude at 1400 m.a.s.1.) [16] has been performed. The study indicated that deflections alter significantly the lateral distribution of muons only at zenith angles $\geqslant 75^{\circ}$. This result, however, would be different if one considers different geomagnetic fields and ground level altitudes [7]. In all cases, for smaller angles, the asymmetry in the particle densities is dominated by both a purely geometrical effect and the effect on the longitudinal development. As a result, the azimuthal symmetry of the spatial distribution is destroyed, changing the dependence on the distance from the shower core, directly affecting the estimate of the energy of the primary particle. Much of the interpretation of EAS phenomenon involves the LDF; in addition to energy determination, the parameter characterizing the actual stage of the EAS development (the so-called age parameter) is expected to contain information about the primary composition as well as the characteristic of high energy interaction.

In this paper, we present an approach to analyze lateral distributions of particles produced by UHECR, which is valid at all zenith angles. We tackle the problem studying, by means of Monte Carlo simulations, the evolution with atmospheric depth of the parameters of semi-analytical lateral structure functions for vertical showers. The results obtained are then used to calculate the corresponding LDF for inclined showers including for the first time both, geometrical and attenuation effects. We also present a comparison of the proposed LDFs with AGASA and Haverah Park experimental data and with MC data including water Cerenkov detector effects.

\section{Analysis of vertical showers}

\subsection{Electron lateral distribution function}

We start our analysis using the lateral structure function derived for a pure electromagnetic cascade by Nishimura and Kamata, and later developed by Greisen $[17,18]$ in the well-known NKG formula. This function has been much used in 
theoretical developments (3D EAS simulation) as well as in the description of experimental results $[19,20]$. The NKG formula is given by:

$$
\begin{aligned}
\rho(r)= & \frac{N_{\mathrm{e}}}{r_{\mathrm{m}}^{2}} \frac{\Gamma\left(4.5-s_{\mathrm{nkg}}\right)}{2 \pi \Gamma\left(s_{\mathrm{nkg}}\right) \Gamma\left(4.5-2 s_{\mathrm{nkg}}\right)}\left(\frac{r}{r_{\mathrm{m}}}\right)^{s_{\mathrm{nkg}}-2} \\
& \times\left(1+\frac{r}{r_{\mathrm{m}}}\right)^{s_{\mathrm{nkg}}-4.5}
\end{aligned}
$$

with $\quad s_{\mathrm{nkg}}=3 /\left(1+\left[2 \ln \left(E_{0} / \epsilon_{0}\right) / t\right]\right) \quad$ and $\quad t=$ $\int_{z}^{\infty} \rho_{\text {atm }}(z) \mathrm{d} z / X_{0}$, where $N_{\mathrm{e}}$ is the total number of electrons, $\epsilon_{0}$ is the critical energy, $E_{0}$ is the primary energy, $r_{\mathrm{m}}$ is the Molière radius and $X_{0}$ is the radiation length in air. The age parameter, $s_{\mathrm{nkg}}$, characterizes the actual stage of the EAS development.

To obtain the lateral distribution of particles it is necessary to consider the multiple Coulomb scattering, which determines the characteristic size of the shower front. The lateral development in electromagnetic cascades in different materials scales well with the Molière radius [21] given by $r_{\mathrm{m}}=E_{\mathrm{s}}\left(X_{0} / \epsilon_{0}\right)$, with the energy scale $E_{\mathrm{s}}=$ $m c^{2}(4 \pi / \alpha)^{0.5}$.

It is straightforward to see that the Molière radius varies with the inverse of the density of the medium.

$r_{\mathrm{m}}=r_{\mathrm{m}}\left(h_{0}\right) \frac{\rho_{\mathrm{atm}}\left(h_{0}\right)}{\rho_{\mathrm{atm}}(h)}=\frac{9.6 \mathrm{~g} \mathrm{~cm}^{-2}}{\rho_{\mathrm{atm}}(h)}$

To study the development of the shower parameters with $t$, we use Monte Carlo simulations of vertical proton induced showers at $10^{19} \mathrm{eV}$ generated using the AIRES code. This program is a realistic air shower simulation system, which includes electromagnetic algorithms [22] and links to different hadronic interactions models. We use in this paper QGSJET [23] for nuclear fragmentation and inelastic collision which is consistent with experimental data [24]. For the highest energy air showers the number of secondaries become so large $\left(>10^{11}\right)$ that it is prohibitive, in computing time and disk space, to follow and store all of them. Hillas [26] introduced a non-uniform statistical sampling mechanism which allows reconstruction of the whole EAS from a small representative fraction of secondaries that are fully tracked. Statistical weights are assigned to the sampled particles to account for the energy of the discarded particles. This technique is called "statistical thinning". The AIRES implementation includes an extended thinning algorithm, which is explained in detail in Ref. [27]. The present work has been performed using, in most cases, an effective thinning level $\epsilon_{\mathrm{th}}=E_{\mathrm{th}} / E_{\text {prim }}=10^{-8}$ which allows the avoidance of unwanted fluctuations and provide a reliable number of particles far from the shower core. All shower particles with energies above the following threshold were tracked: 90 $\mathrm{keV}$ for $\gamma \mathrm{s}, 90 \mathrm{keV}$ for electrons and positrons, 10 $\mathrm{MeV}$ for muons, $60 \mathrm{MeV}$ for mesons and $120 \mathrm{MeV}$ for nucleons and nuclei.

With the particle data recorded, we have evaluated the lateral distribution of electrons and positrons not only at ground altitude, but also at different atmospheric depths $t$. Fits to LDF were performed using Eq. (1) to obtain the corresponding parameters as a function of $t$, in a shower-to-shower analysis. We found that the Molière radius $r_{\mathrm{m}}$ deviates from (2) when using a value of the age parameter fixed to the theoretical prediction value, which is an approximation valid for pure electromagnetic cascades. Undoubtedly, a different age parameter should somehow be used to reproduce showers initiated by hadrons. This point has already been addressed experimentally [28-32,34-36] and extensively studied by several authors (see [36] and references therein).

Our studies of vertical showers confirm, as proposed by [37], that a modified NKG formula should be used. Modifications of the NKG formula have been presented using theoretical considerations [38] as well as parameterizations induced by Monte Carlo simulations . In the first case, the method is subject to several approximations to obtain an analytical expression. The second one relies on Monte Carlo results at ground level yielding average values of the parameters which are then included in the parameterizations. For our purpose it is important to obtain a very general LDF which can be used to describe the behavior of lateral distributions of electrons and positrons for vertical showers, not only at ground level but at any atmospheric depth. We use a NKG function with an age parameter given by 

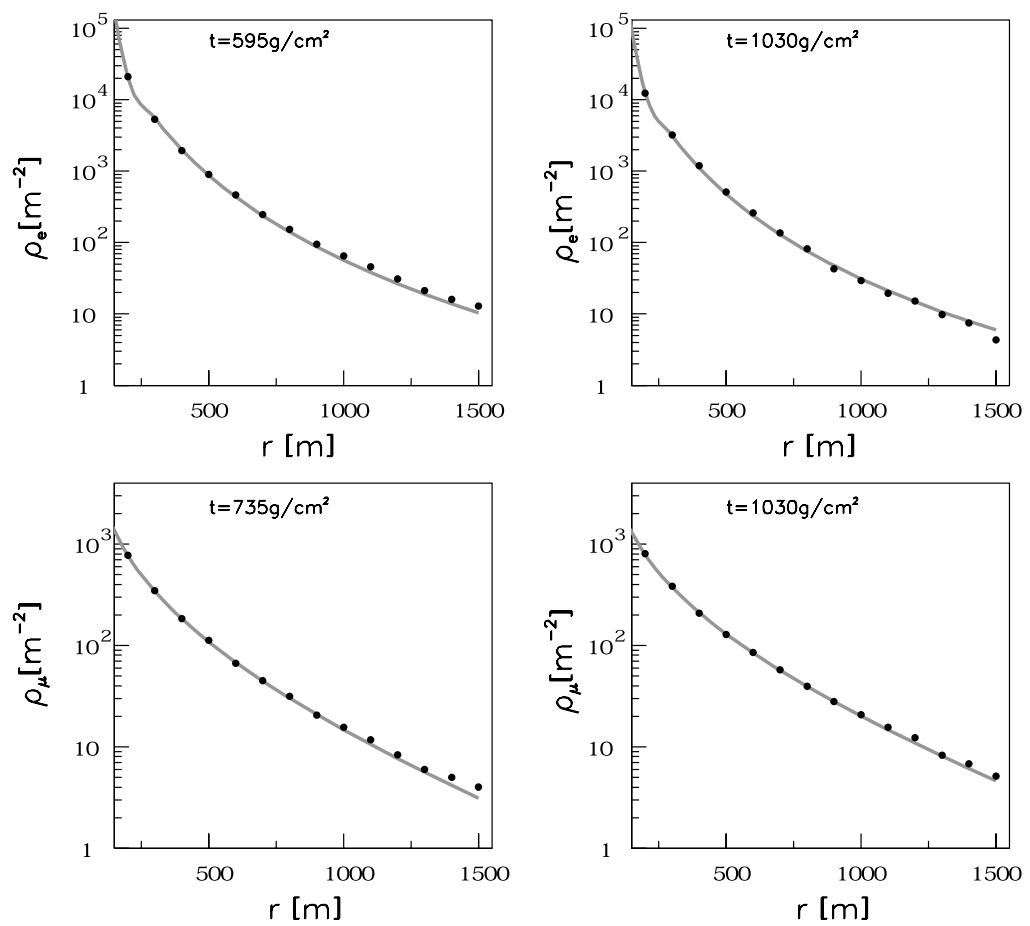

Fig. 1. Electron + position (top) and muon (bottom) lateral distributions of a $10^{19} \mathrm{eV}$ vertical proton shower at different atmospheric altitudes. The solid line indicates the result of the fit using the proposed LDF (see text).

$s=\frac{3}{1+2 \beta / t}$

where $\beta$ takes into account the above mentioned deviations from the theoretical value $s_{\mathrm{nkg}}$. The proposed NKG formula provides a good description of the LDF at all stages of shower development only outside the hadronic core which lies close to the shower axis. It should be mentioned at this point that most UHECR experiments can measure densities only at $r>100 \mathrm{~m}$ from the axis, since in the very dense region near the shower core the particle numbers are so large that array detectors would be saturated for high energy showers and there is rarely a detector there. Fits to Monte Carlo data at $r>100 \mathrm{~m}$ from the shower core were performed with $r_{\mathrm{m}}$ fixed to (2), leaving the overall normalization and $\beta$ as free parameters.

Fig. 1 (top) shows the Monte Carlo $\mathrm{e}^{+} \mathrm{e}^{-}$density distributions corresponding to a single $10^{19} \mathrm{eV}$ proton shower at selected atmospheric depths from the whole set of levels considered. The error bars are included in all cases in the points. Solid lines are the results of the fits. The reduced $\chi^{2}$ are in the range 1-3 with better fits around the position of the shower maximum.

The behavior of the fitted age parameter with atmospheric depth is displayed in Fig. 2. A set of 15 proton initiated showers was used to analyze the natural fluctuations. With different symbols it is shown the average fitted $s$ value at different distances from the shower core; $150 \mathrm{~m}<r<450$ $\mathrm{m}$ (circles), $150 \mathrm{~m}<r<750 \mathrm{~m}$ (squares) and $150 \mathrm{~m}<r<1600 \mathrm{~m}$ (triangles). The error bars, in most cases smaller than the points, represent the natural shower-to-shower fluctuations. (The RMS fluctuations of the mean are always smaller than the symbols.) A remarkable feature is the fact that the whole radial range beyond $150 \mathrm{~m}$ can be described by a unique age parameter independent of $r$. The band in the same figure shows the age parameter calculated using (3) with the assumptions that $\beta$ is given by the position of average shower maximum, $\left\langle X_{\max }\right\rangle=730 \pm 36 \mathrm{~g} \mathrm{~cm}^{-2}$ for 


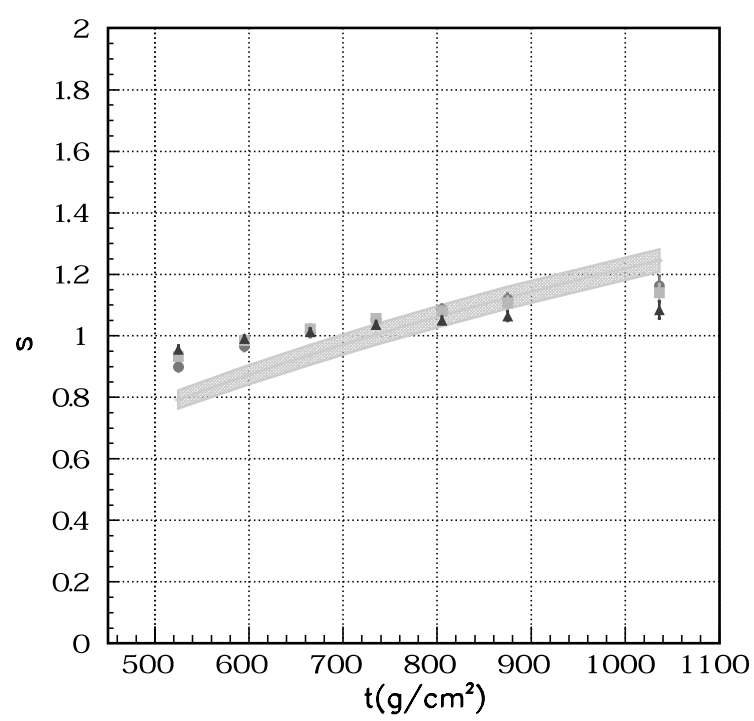

Fig. 2. Atmospheric depth dependence of the average age parameter obtained fitting electron lateral distributions of single showers at different radial distances: $150 \mathrm{~m}<r<450 \mathrm{~m}$ (circles), $150 \mathrm{~m}<r<750 \mathrm{~m}$ (squares) and $150 \mathrm{~m}<r<1600 \mathrm{~m}$ (triangles). Shaded area indicates the expected dependence using a NKG formula with (1).

the shower set used. It is evident that, around the shower maximum the agreement is better and the value of the age parameter is approximately 1 at $X_{\max }$.

The total number of electrons obtained from the fit to each single shower is slightly lower than the actual one. This can be explained due to the fact that the shower size depends on extrapolation of the LDF in the region close to the core, which is not included in the fit.

\subsection{Muon lateral distribution function}

It is well established that at ground level and for large zenith angles the muonic component of an EAS becomes dominant $[7,16]$, reaching a maximum proportion at $75^{\circ}$, after which it decreases slightly [16]. Consequently, the study of muon density distributions at all zenith angles is very important. Several parameterizations have been presented for the muon lateral distributions produced in vertical showers; a NKG-type LDF was empirically derived by Greisen [17]

$$
\begin{aligned}
\rho_{\mu}(r) & =N_{\mu}(t) f_{\mu}(r) \\
f_{\mu}(r, s) & \approx\left(\frac{r}{r_{\mathrm{G}}}\right)^{-0.75}\left(1+\frac{r}{r_{\mathrm{G}}}\right)^{-2.5}
\end{aligned}
$$

fixing $r_{\mathrm{G}}=320 \mathrm{~m}$. Vernov et al. ([39] and references therein) proposed a semi-analytical form of the structure function

$f_{\mu}(r)=C\left(\frac{r}{r_{0}}\right)^{-\gamma} \exp \left(-\frac{r}{r_{0}}\right)$

with $\gamma=0.4$ and $r_{0}=80 \mathrm{~m}$. A similar approach was suggested by Hillas [12]. The slope of the two functions are in very good agreement at intermediate distance, but Vernov distribution is flatter close to the shower core and decreases faster at larger distances. These LDF have been used to fit experimental data. However, neither function seems to reproduce the whole radial range of an EAS. The reason for that is clearly the lack of dependence on shower stage development, using instead fixed parameters in the corresponding structure functions. Very recently, the KASCADE experiment has used a NKG formula to fit muon density distributions. The fits were performed close to the shower core $(r<200 \mathrm{~m})$ with non-conventional $r_{\mathrm{m}}$ and $s$ values [19].

A dependence of the parameters according to the shower age must be expected for the LDF although not precisely the same as the one exhibited by electromagnetic component. Unlike electrons, muons in an air shower are less attenuated and little affected by Coulomb scattering. The muon spread is determined by the direction of emission of the parent particle and hence increases while the shower propagates downwards. A shower age dependence of the muon structure function has been considered in relation with the parameter $\gamma$ (see [40] and references therein).

For the purpose of this paper the Vernov function seems to be a better choice due to its analytical origin. To study the depth dependence of the parameters, we use a modified Vernov distribution with a $\gamma$ parameter dependence with atmospheric depth given by $\gamma=2-s$, with $s$ as in (3) due to the fact that in the radial region smaller than the scale radius, the slopes predicted by both the NKG-like and Vernov distribution are very similar. 
It has been shown $[41,42]$ that, if the parent particles are created with a $p_{\perp}$ distribution: $p_{\perp} / p_{0}$ $\exp \left(-p_{\perp} / p_{0}\right) \mathrm{d} p_{\perp} / p_{0}$, then the Vernov distribution is obtained at ground level, with $r_{0}$ given by

$r_{0}=\frac{2}{3}\left\langle H_{\mathrm{p}}\right\rangle \frac{\left\langle p_{\perp}^{\mu}\right\rangle}{\left\langle E_{\mu}\right\rangle}$,

where $\left\langle p_{\perp}\right\rangle=2 p_{0}$ is the mean transverse momentum, $\left\langle E_{\mu}\right\rangle$ the mean energy of muons and $\left\langle H_{\mathrm{p}}\right\rangle$ the mean height of production.

These approximate expressions can serve to calculate the variation with $t$ of the parameters characterizing the lateral spread. The ratio $\left\langle p_{\perp}^{\mu}\right\rangle /\left\langle E_{\mu}\right\rangle$ can be considered constant while the shower develops [7] and the variation of $r_{0}$ with altitude is only determined by the $t$ dependence of the mean high of origin of muons. These particles are produced in every pion generation and their energy distribution follows that of their parents. Thus, one expects the dependence of $\left\langle H_{\mathrm{p}}\right\rangle$ with $t$ to arise from two sources. The trivial one resulting from the fact that we consider observing levels at different altitudes, which gives a factor $h_{0} \log \left(t / t_{\mathrm{p}}\right)$ assuming an isothermal atmosphere of scale height $h_{0}$; and a dynamical one due to the pion decay process. It is well known that, at a given energy, the pion decay probability decreases with increasing air density. Thus, a characteristic radius $r_{0}(t)$ results,

$r_{0}(t)=\frac{2}{3} \frac{\left\langle p_{\perp}^{\mu}\right\rangle}{\left\langle E_{\mu}\right\rangle} h_{0} \frac{t_{\mathrm{G}}}{t} \log \left(\frac{t}{t_{\mathrm{p}}}\right)$

where $t_{\mathrm{G}}$ is the ground level depth in $\mathrm{g} \mathrm{cm}^{-2}$. Note that at $t=t_{\mathrm{G}}$ Eq. (5) is recovered.

Fits using the modified Vernov function to the muon density distributions obtained from the same Monte Carlo set of showers generated as described in the previous section, were performed with $N_{\mu}, r_{0}$, and $\beta$ simultaneously free. Reduced $\chi^{2}$ of the fits to single showers for all predetermined observing levels are in the range 1-3. Fig. 1 (bottom) displays the $\mu^{+} \mu^{-}$density distributions (points) for a single $10^{19} \mathrm{eV}$ proton shower corresponding to a subset of the atmospheric levels, together with the fits (solid lines). The agreement is evident.

The parameter $\beta$ is approximately constant and much bigger than the shower $X_{\max }$. This feature is somehow consistent with the fact that muons be-

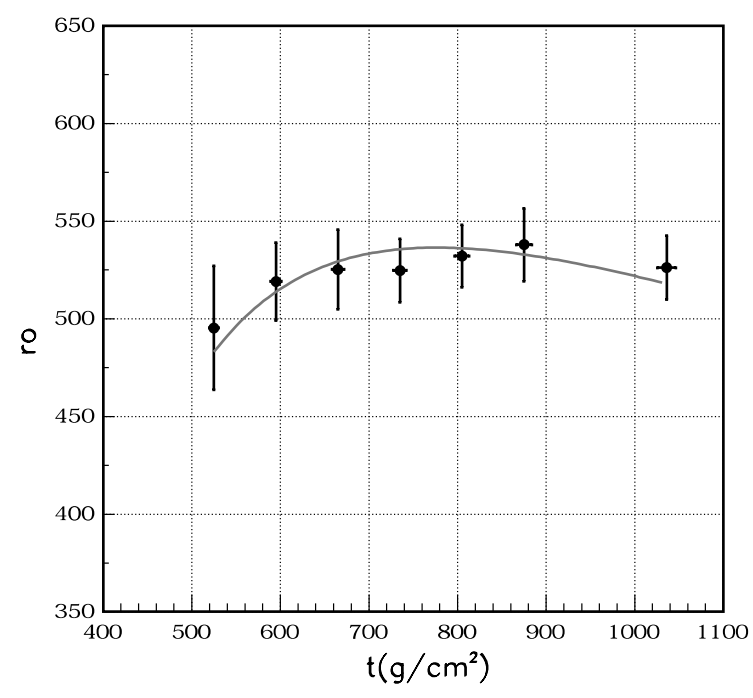

Fig. 3. Atmospheric depth dependence of the average scale radius $r_{0}(t)$ fitting single showers. The error bars correspond to the standard fluctuations. Solid line is the result of the fit using Eq. (6).

come relatively more and more abundant, with only a slight attenuation, as the atmospheric depth increases.

The mean value of $r_{0}$ from the above mentioned set of showers is plotted in Fig. 3, where error bars indicate the natural shower-to-shower fluctuations. The solid line corresponds to a fit using Eq. (6) which gives $t_{\mathrm{p}}$ of the order of $\Lambda_{\mathrm{N}}$, the attenuation length for nucleons in air, as expected. Note that $r_{0}$ is a flat function of $t$ resulting from a compensation of the two factors described above. For inclined showers, the typical slant depth of the shower increases significantly with the zenith angle and the $t$ dependence becomes dominated by the atmospheric density factor.

Fig. 4 displays the age parameters obtained fitting different radial ranges: $100-300 \mathrm{~m}$ (circles), 100-600 m (squares) and 100-1600 m (triangles). The error bars indicate, as in the other cases, the natural shower fluctuations. The curve shows the prediction using (3) and constant value of $\beta=1800 \mathrm{~g} \mathrm{~cm}^{-2}$. The total number of muons $N_{\mu}$ from the fits agrees quite well with the corresponding values registered from the Monte Carlo data, in spite of the fact that the fits are performed in all cases at core distances $r>100 \mathrm{~m}$, implying 


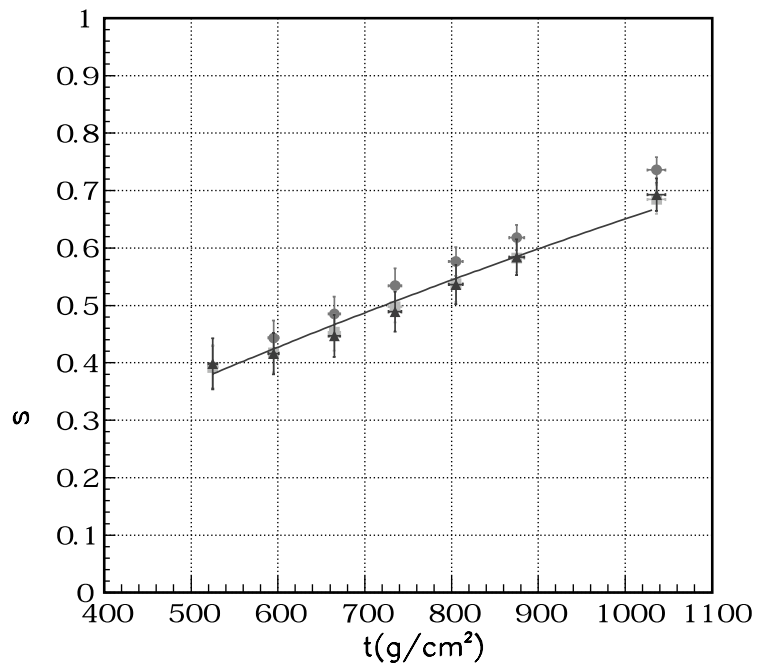

Fig. 4. Atmospheric depth dependence of the average age parameter obtained fitting muon lateral distributions of single showers at different radial distances: $100 \mathrm{~m}<r<300 \mathrm{~m}$ (circles), $100 \mathrm{~m}<r<600 \mathrm{~m}$ (squares) and $100 \mathrm{~m}<r<1600 \mathrm{~m}$ (triangles) The line indicates the fit as described in the text.

an extrapolation of the LDF beyond the range considered.

\section{Inclined showers}

The main goal of the analysis done on vertical shower was to obtain the atmospheric depth dependence of the shower parameters. This result is crucial to properly take into account the effect of the attenuation in the atmosphere observed in inclined showers, as we shall show in what follows. In this section we extend the solutions obtained for vertical showers to inclined ones, considering not only the geometrical effect but also the influence of the attenuation in the atmosphere.

It must be emphasized that the measured data by surface arrays are the ground density of particles and the showers are analysed projecting the ground densities into the shower plane. As mentioned in the introduction, this procedure allows correction of the geometrical effects and it is a good approximation for near-vertical showers. However, it was shown in the Section 2 for vertical showers, that the parameters of the LDF depend on the atmospheric depth $t$. This means that to explain the observed asymmetry, besides the transformation to shower front coordinates, one has to evaluate all parameters of the LDF at the new slant depth $t^{\prime}$

$t^{\prime}=\frac{\int_{z^{\prime}}^{\infty} \rho_{\text {atm }}(z) \mathrm{d} z^{\prime}}{X_{0}}$

with $z^{\prime}$ along the shower axis direction, to include the attenuation effect in the atmosphere.

Actually, the structure of an air shower is quite complex but following [43,44], an inverted cone seems to be a reasonable first approximation. The intersection of an inclined cone with a plane parallel to the ground gives,

$$
\frac{z}{\cos \theta}-L=\left(z^{\prime}-L\right)\left(1-\frac{r^{\prime}}{z^{\prime}-L} \tan \theta \cos \phi\right)
$$

The parameters $L, \theta$ and $\phi$ are indicated in Fig. 5. If the cone angle $\alpha$ is assumed nearly independent of $z$ then, the slant depth $t^{\prime}$ results from:

$\frac{t}{t^{\prime}}=\cos \theta\left(1-\tan \alpha \tan \theta \cos \phi^{\prime}\right)$

Particle density distributions for inclined showers are given by the corresponding electron and muon LDF but evaluated at slant depth $t^{\prime}$ where the dependence on the azimuthal angle is

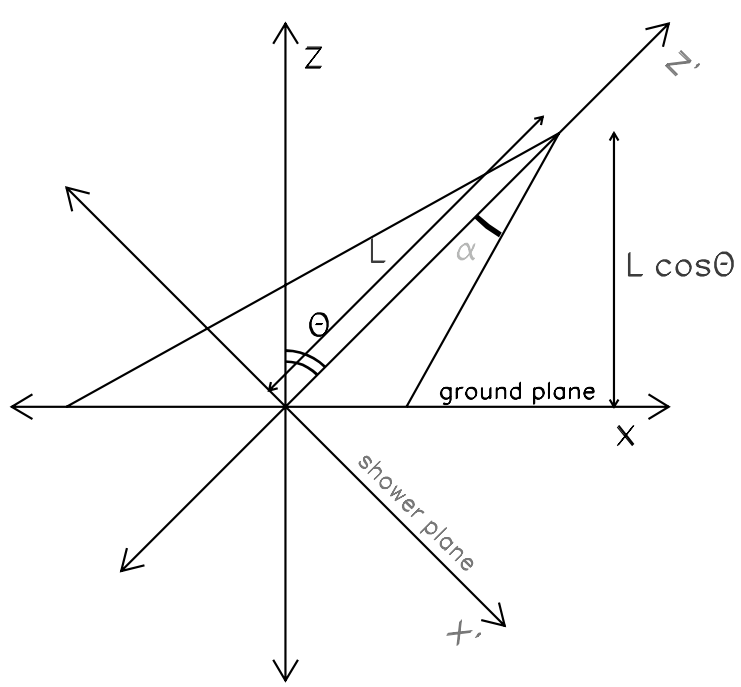

Fig. 5. The intersection of an inclined cone and the ground plane. 
evident. Proton air showers at $10^{19} \mathrm{eV}$ were generated under the same conditions of vertical showers with AIRES + QGSJET at zenith angles between $\theta=10^{\circ}$ and $75^{\circ}$, where the influence of the geomagnetic field is still negligible. The geomagnetic field was then switched off in the simulation. The primary particle arrives at the corresponding zenith angle from $0^{\circ}$ east of the south.

For single showers, two-dimension density distributions $(r-\phi)$ were built at all predetermined observing levels for different particles with bins $100 \mathrm{~m}$ and $30^{\circ}$ wide.

\subsection{Electron lateral and azimuthal distributions}

The distributions were fitted using the modified NKG formula with $s$ (Eq. (3)) including the com- plete zenith angle dependence, with $\alpha, \beta$ and $N_{\mathrm{e}}$ as free parameters. The proposed LDF fits the $\mathrm{e}^{+} \mathrm{e}^{-}$ distributions very well at all observing levels for $\theta \leqslant 60^{\circ}$. At zenith angles greater than $60^{\circ}$ the fits are good only up to a slant depth $t^{\prime} \sim 2000 \mathrm{~g} \mathrm{~cm}^{-2}$. This can be understood in the light of the physics behind air showers induced by nucleons or nucleus. These particles undergo nuclear collisions in the atmosphere. The cascade begins with a hadronic interaction with an increasing number of pions in each generation of particle interactions. The rapid decay of $\pi^{0}$ s into photons feeds the electromagnetic cascade, which finally dissipates $\approx 90 \%$ of the primary energy while charged pion decays produce the muons and neutrinos. The development of the shower reaches a maximum at around $800 \mathrm{~g} \mathrm{~cm}^{-2}$ for vertical $10^{19} \mathrm{eV}$ proton
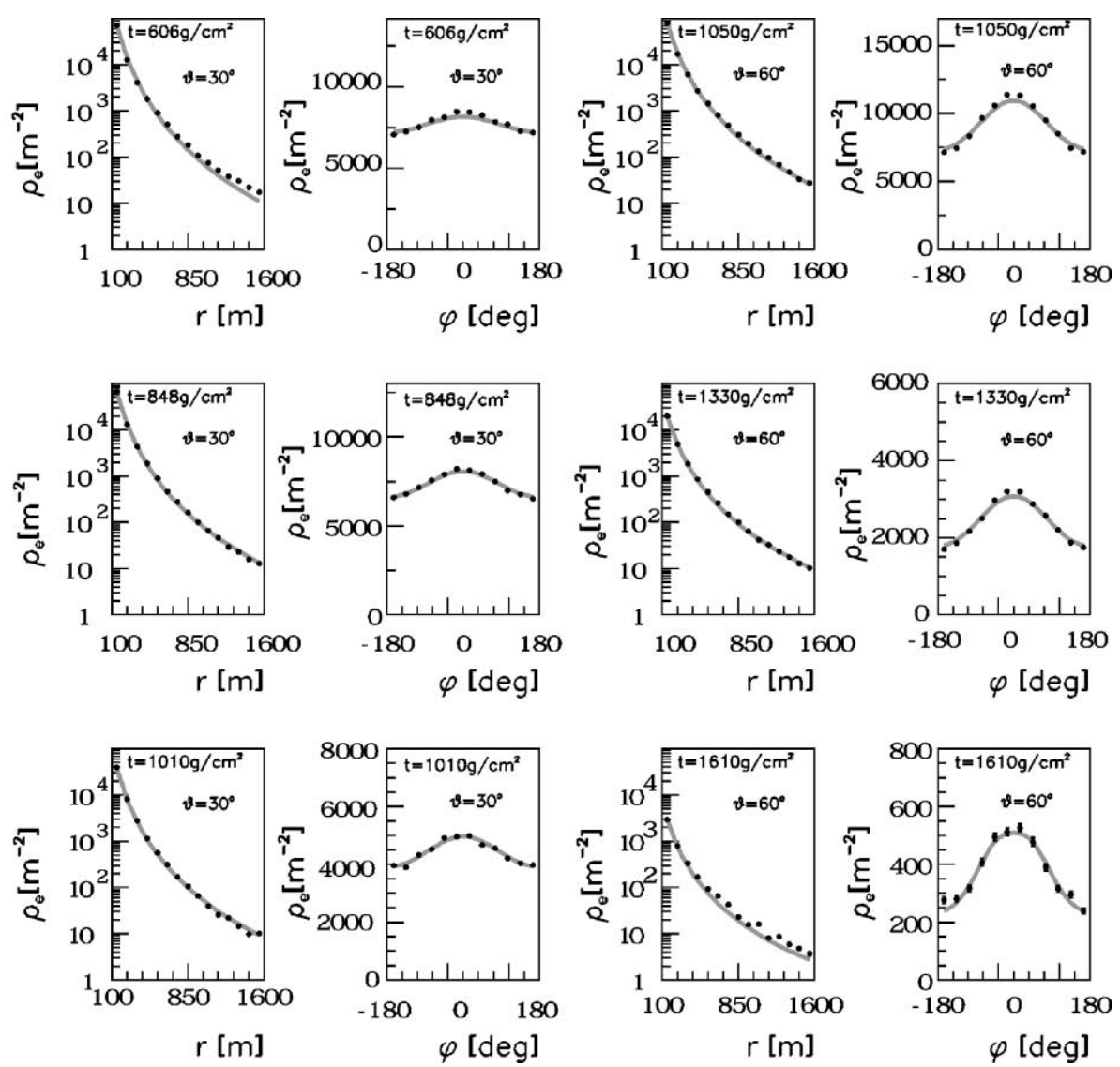

Fig. 6. Projections of two-dimension electron distributions $(r-\phi)$ at different slant depths for a $10^{19} \mathrm{eV}$ proton shower at $30^{\circ}$ and $60^{\circ}$ zenith angles. 
showers. However for inclined showers, the slant depth rises dramatically from $\approx 1000\left(\theta=0^{\circ}\right)$ to $36,000 \mathrm{~g} \mathrm{~cm}^{-2}\left(\theta=90^{\circ}\right)$ at sea level. A detailed study of the characteristics of the muon and electromagnetic component in very inclined showers can be found in Ref. [7]. A standard feature of very inclined showers is that particles at the ground are dominated by the muonic component, while the electromagnetic component is almost completely absorbed due to the greatly enhanced atmospheric slant depth. Beyond $\approx 2000 \mathrm{~g} \mathrm{~cm}^{-2}$ the small electromagnetic cascade is mostly originated from muon decay and knock-on electrons and one cannot expect a NKG distribution for such electrons.

Fig. 6 shows a set of $r$ projection (integrated contents over all $\phi$ ) and $\phi$ projections (integrated contents over the fitted $r$ range) on the shower front from the two-dimension distributions at given atmospheric depths, corresponding to $30^{\circ}$ and $60^{\circ}$ inclined showers. Points with error bars stand for simulated data and curves represent the result of the fit. It is important to note the asymmetry due to atmospheric attenuation effects, higher particle density at $0^{\circ}$ azimuth angle and a depression at $180^{\circ}$. This is evident in the plots showing $\phi$ projections, even for the case $\theta=30^{\circ}$, becoming very important at larger zenith angles. Note also the symmetry in the density at $\pm 90^{\circ}$. It can be seen from the plots that our approach for the LDF predicts the correct dependence for the azimuthal angle distribution. In observations with large zenith angles, the position of the depth of maximum is more distant from the ground with an increasing atmospheric absorption of the electromagnetic component. However, the plots indicate that, even at $\theta<70^{\circ}$ a significant number of electrons can reach the ground level.

Fig. 7 (top) displays the fitted age parameters at selected atmospheric depths for single showers at $0^{\circ}$ (circle), $30^{\circ}$ (square), $50^{\circ}$ (triangle) and $60^{\circ}$ (diamond). When plotting together the results obtained from different showers at different levels, it is evidence that the fitted age parameters overlap smoothly with only small differences due to shower-to-shower fluctuations. This result implies a consistency check and confirms the validity of our approach. With the same aim, the corresponding total number of electrons obtained from the
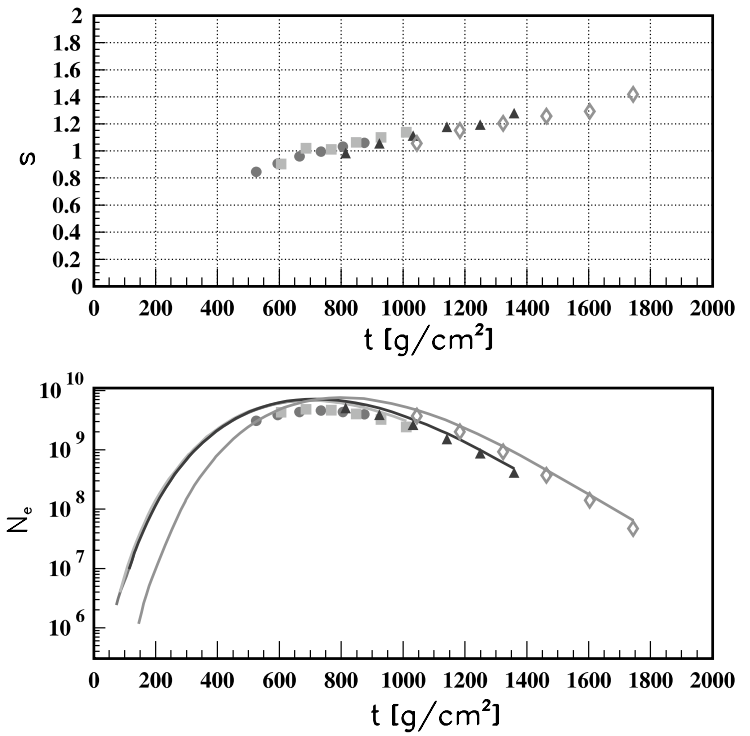

Fig. 7. Upper plot: age parameters at selected atmospheric depth for showers entering at $0^{\circ}$ (circle), $30^{\circ}$ (square), $50^{\circ}$ (triangle) and $60^{\circ}$ (diamond) zenith angles. Shower size obtained from the fits vs slant depth are shown in the lower plot. The actual total particle numbers for each single shower are indicated with solid lines.

AIRES output are plotted in solid lines in Fig. 7 (bottom), along with the fitted shower size $N_{\mathrm{e}}$ (points with same convention than in Fig. 7 (top)) at selected atmospheric depth. It is once more worth mentioning that the curves which correspond to single showers with different incident zenith angles are of course, affected by the fluctuation of the first interaction point. However, the shower size obtained from the fit agrees rather well with the values from the Monte Carlo results, showing the expected longitudinal shower development.

The parameter $\tan (\alpha)$ takes values between 0.15 and 0.2, as expected, since an EAS covers a large area but subtends a small apex angle.

\subsection{Muon lateral and azimuthal distributions}

The same set of generated showers, proton air showers at $10^{19} \mathrm{eV}$ at zenith angles between $\theta=30^{\circ}$ and $70^{\circ}$ with an effective thinning level of $10^{-8}$, was used to analyze the muon component.

Using the procedure described in the previous section for electrons, for each single shower 
two-dimension density distributions $(r-\phi)$ were built at all predetermined observing levels. To perform the fit to the muon densities resulting from the inclined showers, the modified Vernov LDF proposed in Section 2.2 was used. The azimuthal angle dependence was included following the approach described in Section 3. The proposed function successfully fits the Monte Carlo data at all zenith angles. Reduced $\chi^{2}$ are in the range $\approx 1-$ 3. As in the case of electrons, the fitted $N_{\mu}$ and $s$ values are compatible with the expected ones. Fig. 8 shows the radial and azimuthal angle projections of the muon density from two-dimensional fits to the data for $60^{\circ}$ and $70^{\circ}$ zenith angles. The dots stand for the Monte Carlo data and the curves correspond to the fits. It seems worth noting that the asymmetry introduced by the atmospheric attenuation in the lateral distribution is small, mainly at observing levels near the ground.
This feature contrasts with the observed one for electrons, even at lower zenith angles. In previous analysis of quasi-horizontal showers [7], the attenuation effect was neglected. However, as mentioned in the introduction, for showers arriving at $\theta>75^{\circ}$ (with high $\mu$ content at ground level) the lateral asymmetry is dominated by geomagnetic effects. The complex behavior of the muon component due to magnetic field effects was studied in great detail in Ref. [7].

\section{Comparison with experimental LDF}

As mentioned above, to estimate the energy of the primary particle, analysis of the lateral distribution of shower particles at ground are important. Depending on the particle detectors of ground array experiments, different LDF para-
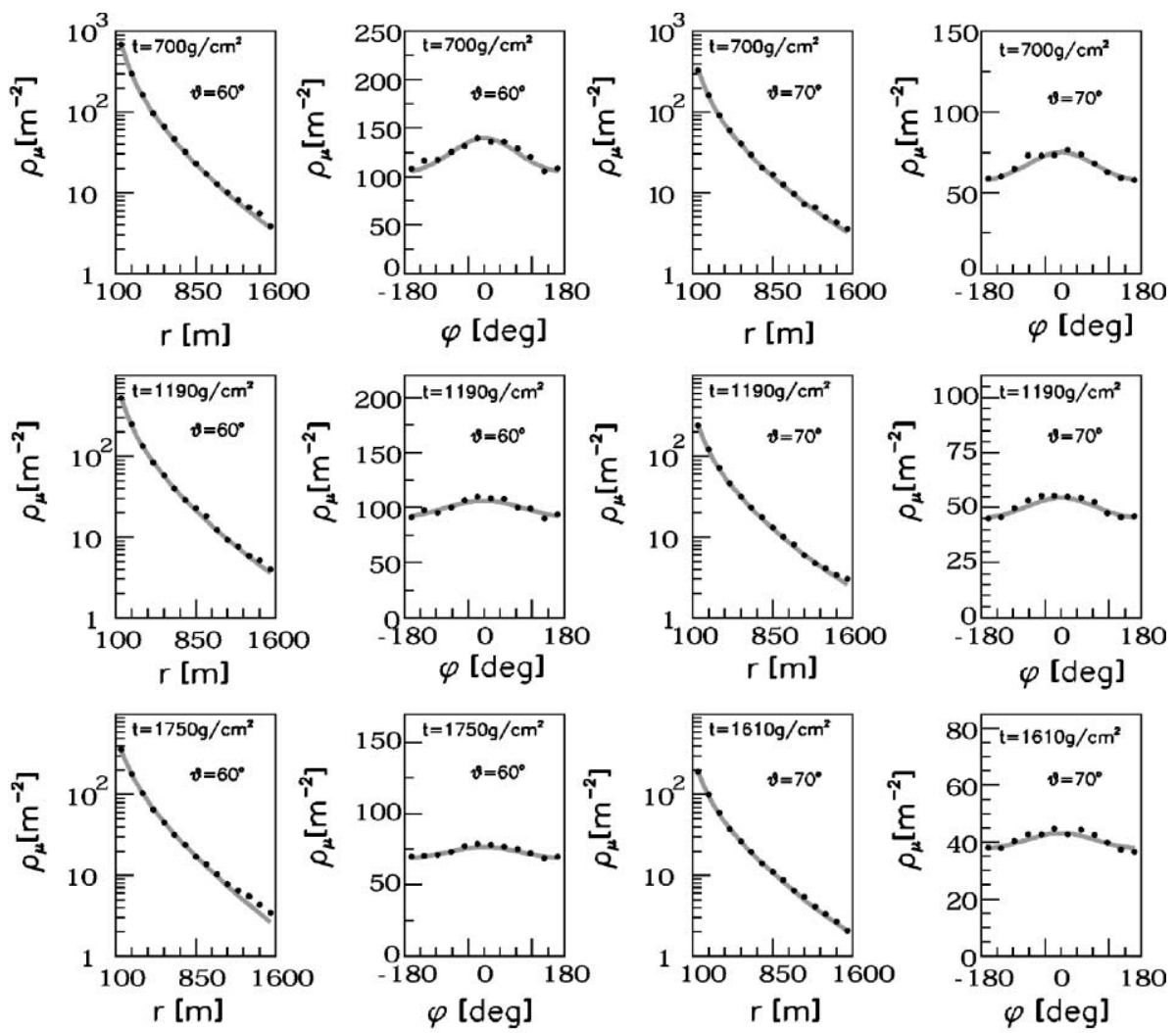

Fig. 8. Projections of two-dimension muon distributions $(r-\phi)$ at different slant depths for a $10^{19} \mathrm{eV}$ proton shower at $60^{\circ}$ and $70^{\circ}$ zenith angles. 
metrizations have been used. The signal measured by plastic scintillators (Volcano Ranch, Yakutsk and AGASA) is dominated by the electromagnetic component of the shower compared with the muon signal. In water Čerenkov detectors (as used in Haverah Park and being implemented for the Pierre Auger Observatory) the electromagnetic and muon components contribute to the total signal. Fig. 9 shows the signal produced by different particles (top) and the ratio of the muon to electromagnetic Čerenkov signal (bottom) measured in units of vertical equivalent muons per $\mathrm{m}^{2}$ for a $10^{19} \mathrm{eV}$ vertical proton shower. It is evident from the plot that the signal changes with distance from the core, being dominated by the muon component far from the shower axis.

The implementation of the method proposed in this paper to obtain the LDF for inclined showers once the appropriate parametrization for vertical showers is found, clearly implies the analysis of the parameters of the corresponding LDF to find their dependence with depth. This specific study has to be performed in all cases using simulations.

In what follows, we compare the parametrizations proposed in Sections 2 and 3 for particle
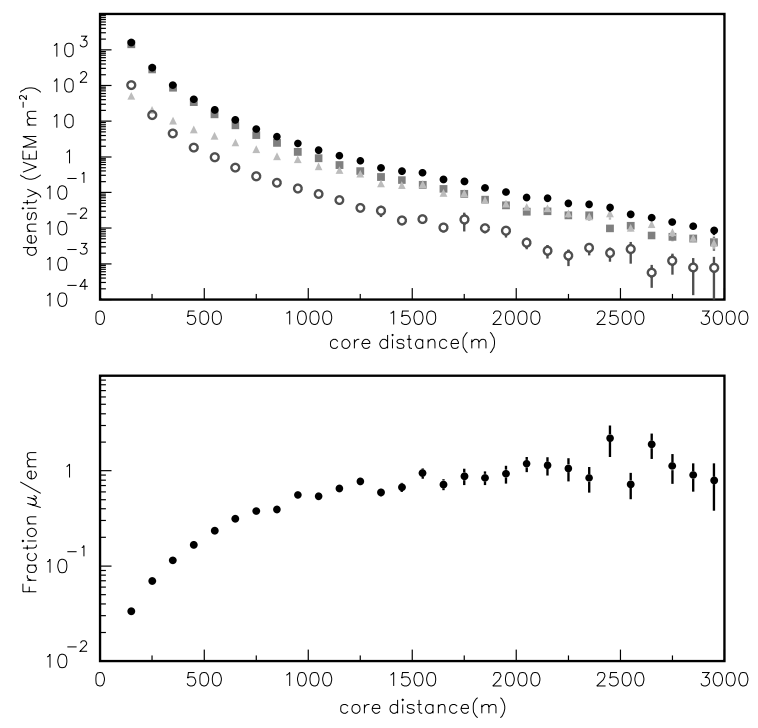

Fig. 9. Top: electron (open circles), gamma (squares), muon (triangle) and total (circles) WCS in VEM m ${ }^{-2}$ vs distance from the shower core for a $10^{19}$ proton vertical shower. Bottom: ratio of muon to electromagnetic signal vs distance from the core. densities with experimental data. It should be stressed that we do not intent to re-analyse the experimental data but rather to investigate to which extent the obtained LDF from pure shower simulations are valid to reproduce experimental data without including additional modifications. In the case that the LDF are suitable to describe experimental data, it would be possible to further check the method presented in Section 3.

For scintillator detectors, generalizations of the NKG distribution have been used to parametrize the observed density with distance. In Fig. 10 we present the experimental result of AGASA for vertical $10^{19} \mathrm{eV}$ showers [45] in the form of their empirical functions for the total signal $S(r)$ and density of muons of threshold energy $1 \mathrm{GeV}$, together with the result of the fit using the parametrizations proposed in this paper. The $S(r)$ signal was fitted with the modified NKG function of Section 2, with the conventional assumption of $r_{\mathrm{m}}=91.6 \mathrm{~m}$. This function can reproduce the AGASA experimental LDF over the core distance $100-2500 \mathrm{~m}$ surprisingly well with a unique value of the age parameter $s=1.05$. If one assumes a

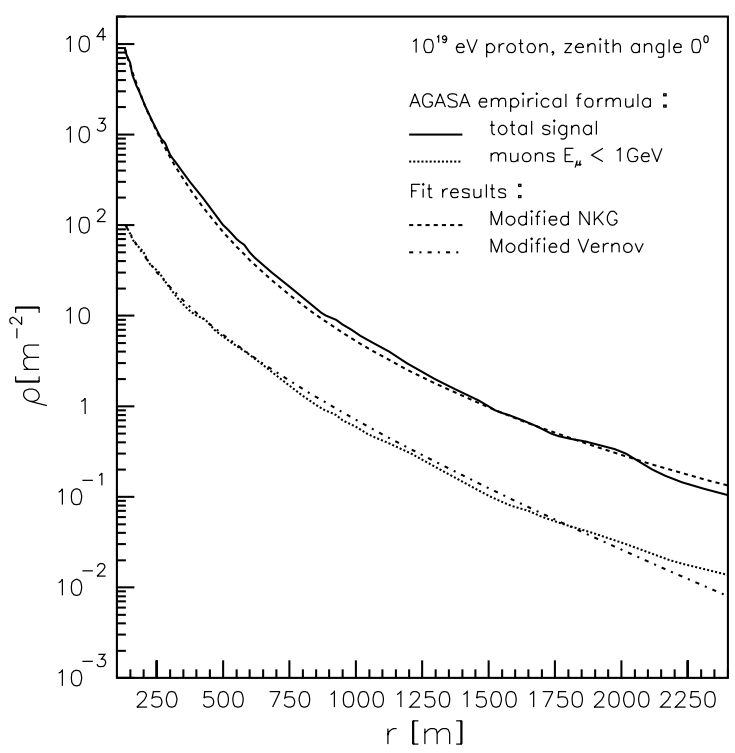

Fig. 10. Lateral distributions of total signal (solid line) and muon (dotted line) determined by AGASA for vertical $10^{19} \mathrm{eV}$ showers, compared with fits using modified NKG (dashed line) and Vernov (dash-dotted line) LDF. 
pure electromagnetic cascade, it is possible to make a very crude estimation of the primary energy from the fitted $\beta$ parameter which results to be $E=1.7 \times 10^{19} \mathrm{eV}$. The value of the signal density at $600 \mathrm{~m}$ from the core with our fit is $41 \mathrm{~m}^{-2}$ to be compare with the one obtained from the AGASA results $S(600)=51 \mathrm{~m}^{-2}$. The average conversion relation for primary proton and iron using two different hadronic interaction models determined by the AGASA experiment is $E(\mathrm{eV})=2.23 \times 10^{17} S_{0}(600)^{1.02}$ [46]. Using this expression we can estimate the energy from our fit to be $E=1.2 \times 10^{19} \mathrm{eV}$.

In addition, we fitted the AGASA muon lateral distribution with the modified Vernov function from Section 2.2 in the range $100-2500 \mathrm{~m}$ with all parameters left free. The result of the fit yields to $r_{0}=436 \mathrm{~m}$. It can be seen that the shape of the fitted LDF is consistent with the empirical AGASA function. The value of $\rho_{\mu}(600)$ from our parameterization is $3.86 \mathrm{~m}^{-2}$ in perfect agreement with the value obtained using the AGASA function.

Due to the lack of published signal densities in the $X-Y$ plane for large zenith angle showers from AGASA experiment, we present here only comparisons with vertical showers. It would be straightforward to extend the analysis to nonvertical showers using the same $\mathrm{LDF}$ and the method we proposed in Section 3. It is worthwhile to remark that most of the experiments have only published cosmic ray showers at small zenith angles.

Water Cerenkov signal (WCS) due to EAS particles at ground includes both electromagnetic particles and muons, and the signal produced in the detectors is the sum of both components. In Fig. 11 we show an extremely large EAS observed by the Haverah Park experiment and their predicted LDF in the regions $r<800 \mathrm{~m}$ and $r>800$ $\mathrm{m}$ as described in [36] (solid lines). The result of the fit using the modified NKG formula from Section 2.1 is also plotted. The Molière radius was fixed to the value calculated using (2) and the mean atmospheric depth of the array; $1018 \mathrm{~g} \mathrm{~cm}^{-2}$. Our LDF reproduces rather well the Haverah Park data in the whole range with age parameter $s=1.24$. From the fitted function a value of

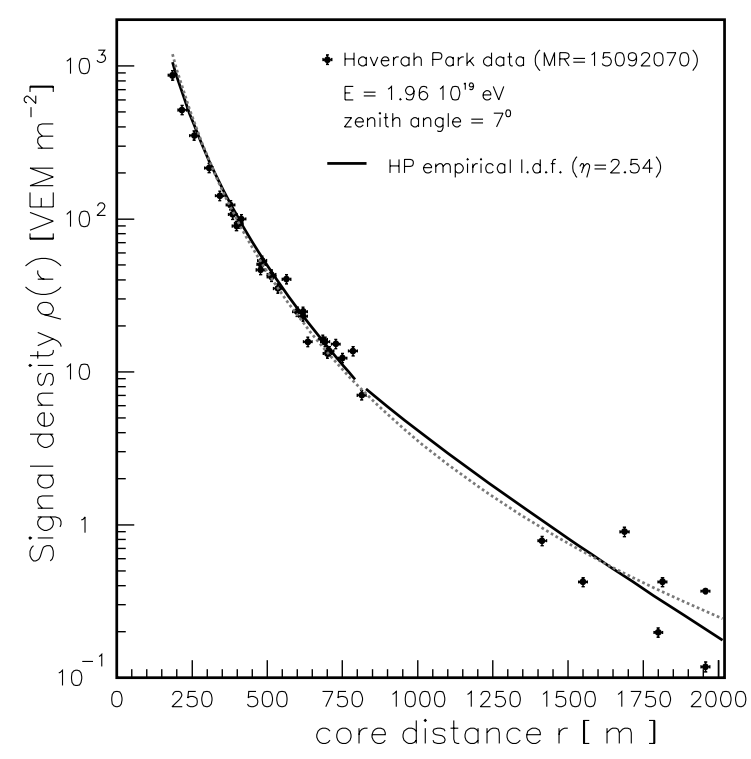

Fig. 11. EAS observed by Haverah Park. The solid line indicates the Haverah Park predicted LDF and dashed-line corresponds to the result of the fit using the modified NKG LDF.

$\rho(600)=23.8$ VEM $\mathrm{m}^{-2}$ was obtained to be compared with the value obtained by Haverah Park $\rho(600)=25.2$ VEM $\mathrm{m}^{-2}$.

To check the validity of our approach for water Čerenkov detectors we have also made estimations of the WCS using a direct conversion procedure that retrieves average signals for every particle hitting the detector [47]. Such averages were evaluated using the AGASIM program [48]. Fig. 12 (top) shows the obtained WCS for all the particles at ground level for a $10^{19} \mathrm{eV}$ vertical shower for distances to the axis beyond $200 \mathrm{~m}$ (dots). The muon threshold energy is $300 \mathrm{MeV}$. The result of the fit using the modified NKG formula leaving all parameters free is superimposed as solid lines, providing a good description of the data $\left(\chi^{2} /\right.$ d.o.f $\left.=26 / 12 \approx 2\right)$. The scale radius determined by the fit is 1.4 times the pure theoretical Molière radius. The age parameter results $s=$ 1.15 , being strongly correlated with $r_{0}$. From the fitted function a value of $\rho(600)=12.7 \mathrm{VEM} \mathrm{m}^{-2}$ was obtained. With this value and the energy calibration formula used to analyse the Haverah Park data [49] $\left(E(E \mathrm{eV})=0.612 \times \rho(600)^{0.99}\right)$, the estimated primary energy results $E_{\mathrm{p}} \approx 0.8 \times 10^{19} \mathrm{eV}$. 

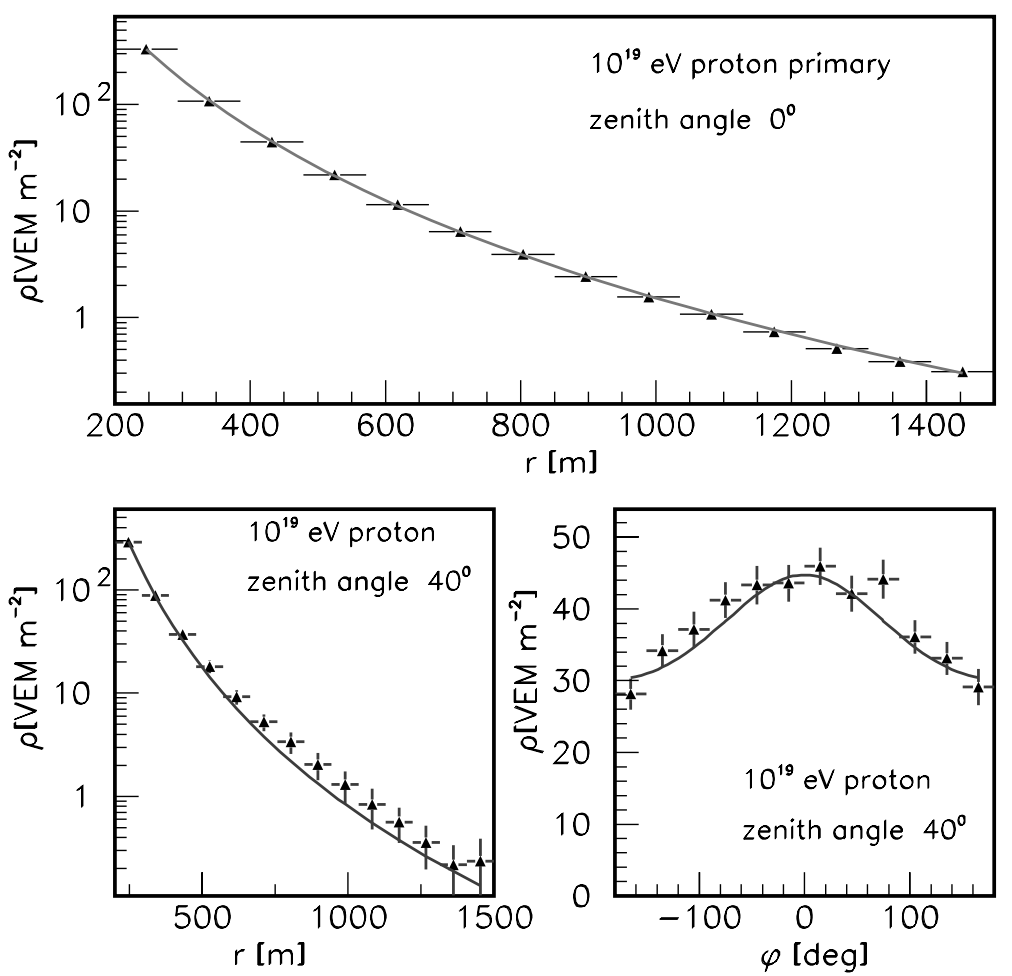

Fig. 12. Top: lateral distribution of signal produced by electrons, muons and photons in a water Čerenkov detector in units of vertical equivalent muons (see text). Bottom: projections of two-dimension WCS distributions $(r-\phi)$ for a proton $40^{\circ}$. Solid line corresponds to the fit performed usig the modified NKG LDF.

Using the parametrization for non-vertical showers derived by application of our method to include the effect of the attenuation in the atmosphere in the modified NKG function, we also fitted the lateral density of WCS at ground for a proton shower at zenith angle of $40^{\circ}$. At this modest zenith angles the average signals estimated as described above are still the appropriate ones. The resulting LDF fits well the data $(\chi 2 /$ d.o.f $=383 / 165=2.3)$ with a fixed value of $r_{0}=1.4 r_{\mathrm{m}}$ as for the vertical showers. The $s$ parameter tends to decrease. It is, however, well known that the shower parameters $s$ and $r_{0}$ are highly correlated. The fitted parameter $\tan (\alpha)$ takes a value of 0.1 in agreement with the expected one. In Fig. 12 (bottom) the radial and azimuthal WCS density projections, measured in VEM $\mathrm{m}^{-2}$, are plotted together with the result of the fitted LDF.

\section{Conclusions}

It is clear that information concerning the lateral distribution functions of particles is very important to determine the characteristics of the ultra high energy cosmic rays. In this paper, we presented a new approach to obtain appropriate parameterizations of LDF, able to reproduce particle density distributions generated for UHECR arriving from any zenith angles.

We started our study with the analysis of vertical showers using modified NKG (Section 2.1) and Vernov (Section 2.2) structure functions for electrons and muons, respectively. A detailed study of the shower parameters with atmospheric depths was performed. The results indicate that a unique age parameter can be used at radial distances greater than $150 \mathrm{~m}$ from the shower core, which is the region of interest for surface arrays. 
The knowledge of the behavior of shower parameters as the cascade of particles develops is crucial to work out the LDF for inclined showers. It is worth noticing that the method presented here it is well suitable to a shower-to-shower based analysis. We paid special attention to the fact that the LDF of particles from inclined cascades must be corrected not only due to geometrical effects but also for the longitudinal attenuation. This latest, usually neglected or considered in a very simple manner in the experimental analysis, is surprisingly large for electron distributions, even at moderate zenith angles where the amount of electrons reaching ground level dominates. Thus, data analysis assuming that the lateral distributions depend only on the distance from the shower axis might yield to biased results if the atmospheric attenuation effect is not considered at all. Muon distributions are also affected by attenuation effects, although to a less extent. The asymmetry at very large zenith angles in this case is dominated by geomagnetic effects.

The obtained lateral distribution functions fit well the generated data at all observing levels along the longitudinal shower paths. The very good agreement of the theoretical distributions for inclined showers obtained using our method with the radial, and more important, the azimuthal angle distributions, provides strong supporting evidence for the validity of this approach. In connection with this result we have checked our method with the signal detected by ground array detectors. We have shown that if the signal produced in water Cerenkov tanks is parametrized by a modified NKG function, then the application of our method is straightforward and the corresponding LDF for inclined showers reproduces the experimental density distributions rather well.

A final comment on the method proposed is well worth making here: several present experiments use both NKG and Vernov type LDF. The application of our method would allow them to include high zenith angle showers in their analysis, what implies a significant increase of their acceptance. Future experiments will also benefit from the analysis of showers arriving from any direction.

\section{References}

[1] N. Hasashida et al., Phys. Rev. Lett. 73 (1994) 3491.

[2] M.A. Lawrence, R.J.O. Reid, A.A. Watson, J. Phys. G 17 (1991) 733.

[3] D.J. Bird et al., Astrophys. J. 441 (1995) 144.

[4] AGASA Collaboration, N. Sakaki et al., Proc. 27th ICRC, 2001, p. 333.

[5] A new cosmic ray spectrum as recorded by the HiRes Collaboration was presented during the 27th ICRC, Hamburg, Germany, August 2001.

[6] M. Nagano, A.A. Watson, Rev. Mod. Phys. 72 (2000) 689.

[7] M. Ave, R.A. Vazquez, E. Zas, Astropart. Phys. 14 (2000) 91;

M. Ave, J.A. Hinton, R.A. Vazquez, A.A. Watson, E. Zas, Astropart. Phys. 14 (2000) 109.

[8] M. Ave, J.A. Hinton, R.A. Vazquez, A.A. Watson, E. Zas, Phys. Rev. Lett. 85 (2000) 2244.

[9] Auger Collaboration, Pierre Auger Project Design Report (2000) Fermi National Accelerator Laboratory. Available from www.auger.org/admin.

[10] K.S. Capelle et al., Astropart. Phys. 8 (1998) 321.

[11] X.Bertou et al., astro-ph/0104452.

[12] A.M. Hillas et al., Proc. 11th ICRC, Budapest, 1969, Acta Physica Academiae Scietiarium Hungariae, vol. 29 (Suppl.) 3, 1970, p. 533.

[13] D. Andrews et al., Proc. 11th ICRC, Budapest, 1969, Acta Physica Academiae Scietiarium Hungariae, vol. 29 (Suppl.) 3, 1970, p. 337.

[14] E.E. Antonov et al., JETP Lett. 69 (1999) 650; JETP Lett. 68 (1998) 185.

[15] A.A. Ivanov, V.P. Egorova, V.A. Kolosov, A.D. Krasilnikov, M.I. Pravdin, I.E. Sleptsov, JETP Lett. 69 (1999) 288.

[16] A.N. Cillis, S.J. Sciutto, Astropart. Phys. 14 (2001).

[17] K. Greisen, Ann. Rev. Nucl. Sci. 10 (1960) 63.

[18] K. Kamata, J. Nishimura, Prog. Theor. Phys. 6 (Suppl.) (1958) 93.

[19] T. Antoni et al., Astropart. Phys. 14 (2001) 245.

[20] M. Nagano, Y. Hatano, T. Hara, N. Hayashida, K. Kamata, T. Kifune, G. Tanahashi, S. Kawaguchi, J. Phys. Soc. Jpn. 53 (1984) 1667.

[21] Particle data group, Eur. Phys. J. C 15 (2000) 170.

[22] A.M. Hillas, Proc. 16th ICRC, Tokyo, Japan 8 (1979) 7; A.M. Hillas, Proc. 17th ICRC, Paris, France 8 (1981) 183.

[23] N.N. Kalmykov, S.S. Ostapchenko, Phys. Atom. Nucl. 56 (1993) 346;

Yad. Fiz. 56 (1993) 105;

N.N. Kalmykov, S.S. Ostapchenko, A.I. Pavlov, Bull. Russ. Acad. Sci. 58 (1994) 1966.

[24] Kascade Collaboration, D. Heck, Proc. 27th ICRC, Hamburg, 2001.

[26] A.M. Hillas, Nucl. Phys. B (Proc. Suppl.) 52B (1993) 29; A.M. Hillas, Proc. 19th ICRC, La Jolla, vol. 1, 1985, p. 155 . 
[27] S.J. Sciutto, astroph/9911331, 1999.

[28] J. Linsley, Proc. 13th ICRC, Denver, vol. 5, 1973, p. 3212.

[29] C. Aguirre et al., Proc. 13th ICRC, Denver, vol. 4, 1973, p. 2592.

[30] N.A. Porter, Proc. 13th ICRC, Denver, vol. 5, 1973, p. 3657.

[31] S. Kawaguchi, K. Suga, H. Sakuyama, Proc. 14th ICRC, Munich, vol. 8, 1975, p. 2826.

[32] M. Nagano et al., J. Phys. Soc. Jpn. 53 (1984) 1667.

[34] S. Yoshida et al., J. Phys. G: Nucl. Phys. 20 (1994) 651.

[35] A.V. Glushkov et al., Proc. 25th ICRC, Durban, vol. 6, 1997, p. 233.

[36] R.N. Coy et al., Astropart. Phys. 6 (1997) 263.

[37] M.F. Bourdeau, J. Capdevielle, J. Procureur, J. Phys. G: Nucl. Phys. 6 (1980) 901.

[38] A.V. Plyasheshnikov, A.A. Lagutin, V.V. Uchaikin, Proc. 16th ICRC Kyoto, vol. 7, 1979, p. 1.

[39] S.N Vernov, G.B. Khristiansen, A.T. Abrasimov, V.B. Atrashkevitch, I.F. Beljaeva, G.V. Kulikov, K.V. Mandri- tskaya, K.B. Solovjeva, B.A. Khrenov, Can. J. Phys 46 (1968) s197.

[40] S. Alessio, H. Bilokon, B. D’Ettore Piazzoli, G. Mannochi, P. Pichi, K. Sitte, Nuovo Cimento 3 (1980) 573.

[41] G.F. Bosia, L. Briatore, K. Sitte, Nuovo Cimento 12 (1972) 1025 .

[42] L. Bergamasco, M. Castagnoli, M. Dardo, B. D'Ettore Piazzoli, G. Mannochi, P. Pichi, R. Visentin, K. Sitte, Nuovo Cimento 34 (1976) 613.

[43] C. Pryke, Auger technical note GAP-98-034, 1998. Available from www.auger.org.

[44] M.T Dova, L.N. Epele, A. Mariazzi, Proc. 26th ICRC, vol. 1, 1999, p. 478.

[45] M. Nagano, D. Heck, K. Shinozaki, N. Inoue, J. Knapp, Astropart. Phys. 13 (2000) 277-294.

[46] N. Sakaki et al., Proc. 27th ICRC, 2001, p. 380.

[47] S.Sciutto, private communication.

[48] C. Pryke, Pierre Auger technical note GAP-97-005 (1997), available elctronically at www.auger.org.

[49] M. Ave et al., Proc. 27th ICRC, 2001, p. 381. 